\title{
An Assessment of Food Safety Knowledge and Practices in the Ghanaian Hospitality Industry
}

\author{
Stephen T. Odonkor ${ }^{1}{ }^{1}$ and Carolyn J. A. Odonkor ${ }^{2}$ \\ ${ }^{1}$ School of Public Services and Governance, Ghana Institute of Management and Public Administration, Accra, Ghana \\ ${ }^{2}$ Young African Leaders Initiative, Ghana Institute of Management and Public Administration, Accra, Ghana \\ Correspondence should be addressed to Stephen T. Odonkor; stodonkor@gimpa.edu.gh
}

Received 7 December 2019; Accepted 28 April 2020; Published 13 May 2020

Academic Editor: Luis Patarata

Copyright (c) 2020 Stephen T. Odonkor and Carolyn J. A. Odonkor. This is an open access article distributed under the Creative Commons Attribution License, which permits unrestricted use, distribution, and reproduction in any medium, provided the original work is properly cited.

\begin{abstract}
Food safety is critical for public health and economic development. The hospitality industry offers a variety of food services, led by food handlers with diverse background. The aim of this paper is to evaluate food safety knowledge and practices among food handlers in the Ghanaian hospitality sectors, with the purpose of informing public policy and improving food safety. A crosssectional survey study was conducted between February and May 2019. Data were obtained from food handlers working in the Ghanaian hospitality industry $(N=306)$. The findings of the study indicate that most $(52.9 \%)$ respondents who work in restaurants had knowledge about food safety principles. Lack of training and education was identified by $63.1 \%$ of the respondents as a major barrier to food safety. Interestingly, $75.8 \%$ of the respondents indicated that food safety practice is not the responsibility of the kitchen staff only. Diarrhea (35.95\%), cholera (35.62\%), and typhoid (27.42\%) were identified as major diseases associated with improper handling of food. Only $65.69 \%$ of the respondents undergo annual medical screening at their workplaces. There is correlation between food safety practice and variables such as marital status, type of residence, age, and education level. Educational campaigns coupled with strict law enforcement should be put in place to ensure that food safety rules and guideline are effectively implemented in the hospitality food industry. Training programs should be organized for food vendors and other stakeholders in the food industry as well. Finally, there should be proactive supervision by local government officials to ensure that good sanitation and hygienic conditions are maintained within the food sectors.
\end{abstract}

\section{Introduction}

The Ghanaian hospitality industry is one of the major anchors of the nation's economy [1]. It consists of hotels, restaurants, colonial forts and castles, and game reserves, among others. A key feature of these tourist destinations is the various food services they offer. In the past few years, the industry has experienced growth due to increased investments and consistent patronage from local and international visitors alike [2]. The hospitality industry in Ghana received $1,316,000$ tourists in 2016, generating an amount of $3.7 \mathrm{M}$ Ghanaian cedis (GHC) $[1,2]$. Apart from the attractive and adventurous sceneries provided by on-site tourist destinations, food delicacies made available at these tourist sites are a vital selling point of the industry. Unsafe food can affect the reputation and patronization in the hospitality industry as it affects choice of eatery [3].

Food safety issues have been a worldwide problem for a long time now. Large population of people are affected by foodborne and health related complications annually [4]. Hence, attention has been given to the implementation of food safety practices in most nations across the globe. The greatest impact of foodborne diseases has been reported in Africa [5]. In Ghana it is estimated that at least 1 out of 40 people are affected with foodborne diseases, which costs government 69 million dollars annually [6]. It is believed that this figure could be higher or worse [7].

To curb or minimize the devastating effects of foodborne illnesses, there is a need to ensure that food safety practices are effectively employed particularly in the hospitality 
industry. Food safety practices can be regarded as activities and procedures involved in ensuring that food that is consumed does not cause harm but accomplishes its desired purpose [6]. In Ghana, safety practices have become relevant in the hospitality industry due to the increasing inflow of tourists coupled with people's continuous demand for food outside their homes which is synonymous with modern lifestyle trends. It is quite obvious that modern lifestyles have propelled most people in the working class, especially urban dwellers, to depend on nourishment outside home. Such people depend on the hospitality industry for breakfast, lunch, supper, and even late-night snacks [8]. They, like tourists, are therefore equally vulnerable to incidences of food poisoning. It is worth noting that in large scale cooking such as that evident in the hospitality industry, food is manipulated by a lot of persons, which increases the plausibility of food contamination [9].

Despite the seemingly increasing risks of foodborne diseases, studies to investigate food safety practices in Ghana's hospitality industry have not been extensive enough. Furthermore, ever since the adoption of the national food safety policy by the Food and Drugs Authority in 2015, official reports or research to access its implementation seems to be elusive. Moreover, several related studies available are narrowed to specific food centers which barely highlight the hospitality industry. For instance, various studies conducted [10-13] were mostly focused on street food vendors, which constitute a meager fraction of the many food sectors which serve the hospitality industry. This leaves a wide gap in food safety practice studies in Ghana's hospitality industry. The present study will fill this gap. It will also be of valuable use to policymakers and practitioners in the hospitality industry, thus aiding in formulating and enforcing regulations, reforms, and activities that will ensure safety food practices in the hospitality industry. The aim of this paper is to evaluate food safety knowledge and practices among food handlers in the Ghanaian hospitality sectors, with the purpose of informing public policy and decision making and improving food safety.

\section{Materials and Methods}

2.1. Description of the Study Location. The study was conducted in the Central Region of Ghana (Figure 1), which lies on the south-eastern part of the country. It is bordered by the Ashanti and Eastern Regions to the north, Greater Accra Region to the east, Western Region to the west, and the Gulf of Guinea to the south. The region occupies a total land area of 9,826 sq. $\mathrm{km}$. The region has 17 administrative districts. The regional capital is Cape Coast, which is the former national capital. It has a population density of 220 people per sq. $\mathrm{km}$. The region is the hub of tourism in Ghana. It has tourist attractions such as forts, castles, and beaches stretched along the Central region's coastline. The famous Kakum National Park, which is endowed with different and rare species of wildlife including 40 species of mammals, over 500 species of butterflies, and a wide array of birds, can be found in the region.
2.2. Research Design and Sample Size. The study employed a cross-sectional design to obtain quantitative data through a pretested questionnaire. The study population included food handlers in the Ghanaian hospitality industry such as hotels, restaurants, fast food joints, and chop bars. The sample size was determined using Miller and Brewer's [14] mathematical formula $\left(n=N / 1+\mathrm{N}(\alpha)^{2}\right)$ for estimating single proportions. In the formulae, $n$ is the sample size, $N$ is the total population, and $\alpha$ is the margin of error. The standard normal deviation was set at a 95\% confidence level, prevalent with the allowable margin of error of 0.08 . The minimum sample size increased and rounded up when $10 \%$ of the calculated, minimum sample size was added for nonresponse or inappropriately filled or missing questionnaires since the questionnaires were interviewer administered.

2.3. Sampling Technique and Study Duration. Data were obtained from a survey of food handlers $(N=306)$ from February 1, 2019, to May 31, 2019. Questionnaires were selfadministered and took an average of 28 minutes to complete. The study utilized a stratified sampling technique to obtain the required number of respondents from each of the four (4) categories of food handlers. Thus, in selecting the respondents, sampling proportionate to size was used to determine the number of respondents to be interviewed from each category of food handlers in the hospitality industry. All adults, 18 years old and above, present in the demarcated category were considered for the study.

2.4. Survey Subjects and Technique. The study utilized a stratified sampling technique. The sector was demarcated into 4 categories: hotels, restaurants, fast food joints, and "chop bars". Therefore, in selecting the respondents for the survey, a sampling proportionate to size was utilized to determine the number of respondents to be interviewed from each category. All adults, 18 years old and above, present in the demarcated category were considered for the study.

2.5. Survey Content. The constructs in the questionnaires were informed by literature with respect to acceptable food safety practices. After each day's interviews, field inspection of questionnaire data was done. This allowed for immediate verification and correction of errors that were identified.

The final survey instrument comprised 34 questions in four thematic areas: sociodemographics (8 items), knowledge about food safety (10 items), respondents' attitudes to food safety (8 items), and food safety practices (8 items). Six experts in social sciences measurement and evaluation determined face validity of the instrument. The average overall face validity was equal to $95 \%$. The study used Cronbach's alpha test for reliability testing, which yielded a reliability coefficient of 0.8 . The Cronbach's alpha test assesses the internal consistency of a set of scale items to ensure that they are all consistent in measuring the same attributes under study. 


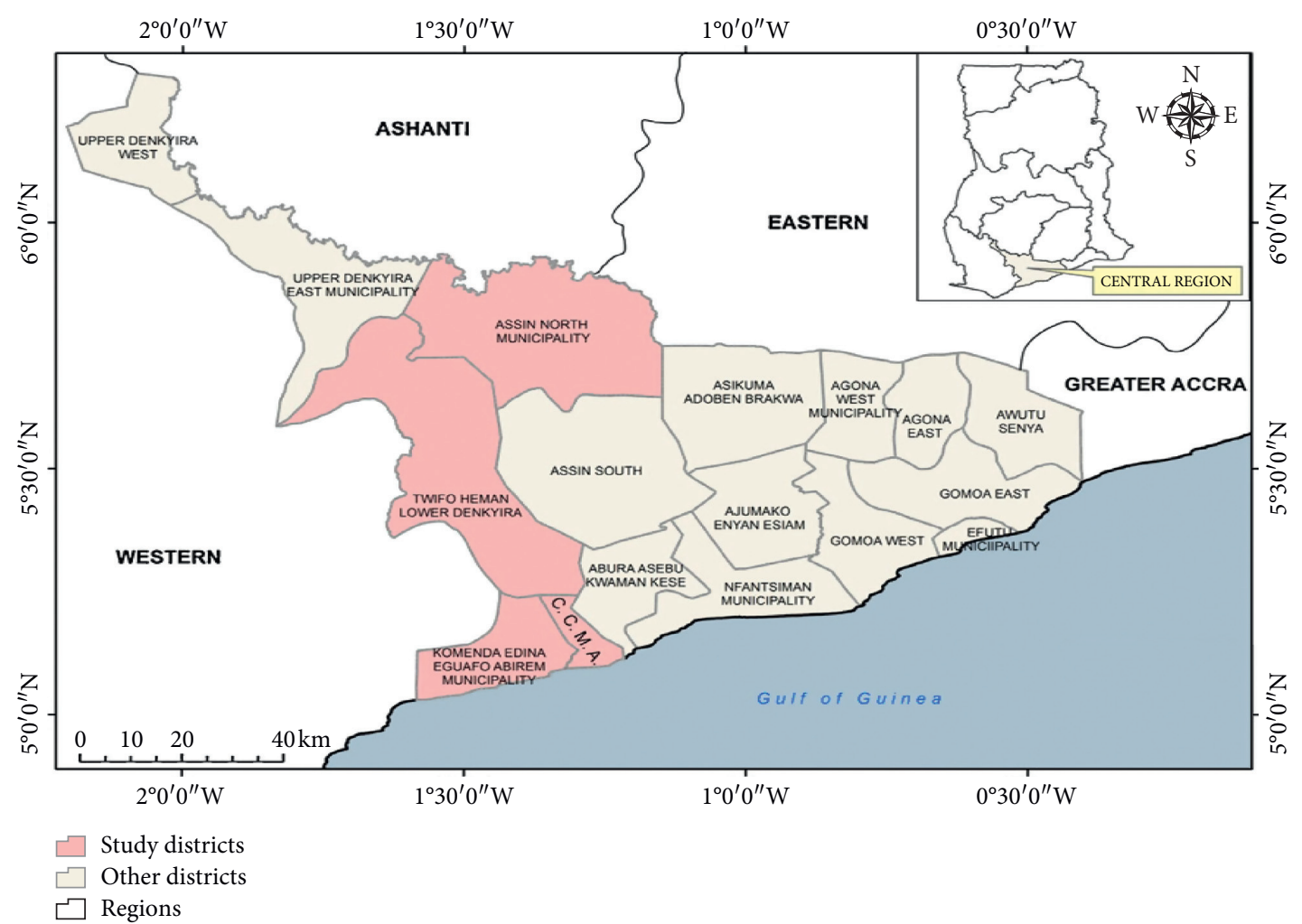

Figure 1: Map of Central Region of Ghana.

2.6. Ethical Consideration. Both verbal and written consent were sought from the respondents before data was obtained. Adequate information was provided to the respondents with regard to the aims of the study. It was made clear to the respondents that their participation was voluntary and that they were at liberty not to participate. They also were assured of confidentiality. All respondents' personal identifiers were deleted from summarized data, ensuring confidentiality. Ethical clearance was obtained from the Ethics Review Committee (ERC) of GIMPA School of Public Service and Governance.

2.7. Statistical Analysis. Data obtained from the questionnaires were coded and analyzed with Statistical Package for the Social Sciences (SPSS) version 23. Discrete variables like gender and educational status were described using frequencies and percentages. Bivariate relationships were analyzed using Chi-Squared $\left(\chi^{2}\right)$ test or Cramer's V exact test. All statistical tests employed in this study were two-tailed and were considered to be significant when the $P$ value is less than the alpha $(P<0.05)$.

\section{Results}

3.1. Demographic Characteristics. Table 1 represents the demographic characteristics of the respondents. There were more $(78.4 \%)$ male respondents in the study than females (21.6\%). In terms of religious background of the respondents, the majority (72.9\%) were Christians. Regarding age category, $55.2 \%$ of the respondents were in the $21-30$ age group, followed by those in the age group of 20 years and below constituting $18.6 \%$. The majority (58.2\%) of the respondents were single, followed by $32.7 \%$ who were married. In terms of the educational background of the respondents, most $(40.5 \%)$ respondents were SHS certificate holders, followed by $26.1 \%$ with vocational level qualification. The majority $(77.1 \%)$ of the respondents were residents in urban areas, whiles $22.9 \%$ were from rural areas.

3.2. Respondents Knowledge of Food Safety Principle. Table 2 shows respondents knowledge of food safety, and HACCP. Most (52.9\%) of the respondents who work in restaurants had knowledge about food safety principles. This was followed by respondents in the fast food industry, where it was observed that $20 \%$ had knowledge about food safety. Interestingly only $11.4 \%$ of respondents from hotels had knowledge about food safety. In terms of the respondent's knowledge about HACCP, it was observed that $31.7 \%$ of respondents from chop bars had knowledge of HACCP, yet this was at variance with the percentage of respondents from the same category who had knowledge about food safety.

Figure 2 shows the respondents perception of diseases associated with improper handling of food. It can be seen from the graph that $35.95 \%$ of the respondents indicated that diarrhea is a disease associated with improper handling of food. This is followed by $35.62 \%$ respondents indicating that cholera is associated with improper handling of food. It is worth noting that $0.98 \%$ of respondents took the view that AIDS is associated with improper handling of food. 
TABLE 1: Demographic characteristics of the respondents.

\begin{tabular}{|c|c|c|c|c|}
\hline Variable & Male $N(\%)$ & Female $N(\%)$ & Total N (\%) & Significance \\
\hline \multicolumn{5}{|l|}{ Religion } \\
\hline Christianity & $48(15.7)$ & $157(57.2)$ & $223(72.9)$ & $\chi^{2}=6.649$ \\
\hline Traditional & $12(3.9)$ & $59(19.3)$ & $71(23.2)$ & $\stackrel{\imath}{P}=0.036$ \\
\hline Other & $6(2.0)$ & $6(2.0)$ & $12(3.9)$ & Cramer's V $=0.036$ \\
\hline Total & $240(78.4)$ & $66(21.6)$ & $306(100)$ & \\
\hline \multicolumn{5}{|l|}{ Ethnicity } \\
\hline Twi & $18(5.9)$ & $45(14.7)$ & $63(20.6)$ & \\
\hline Ewe & $11(3.6)$ & $32(10.5)$ & $43(14.1)$ & $\chi^{2}=4.606$ \\
\hline $\mathrm{Ga}$ & $9(2.9)$ & $38(12.4)$ & $47(15.4)$ & $\stackrel{n}{P}=0.466$ \\
\hline Fante & $26(8.5)$ & $110(35.9)$ & $136(44.4)$ & Cramer's V $=0.466$ \\
\hline Frafra & $2(0.7)$ & $9(2.9)$ & $11(3.6)$ & \\
\hline Other & $6(2.0)$ & $6(2.0)$ & $12(3.9)$ & \\
\hline Total & $66(21.6)$ & $240(78.4)$ & $1306(100)$ & \\
\hline \multicolumn{5}{|l|}{ Age } \\
\hline 20 and below & $10(3.3)$ & $47(15.4)$ & $57(18.6)$ & \\
\hline $21-30$ & $33(10.8)$ & $136(44.4)$ & $169(55.2)$ & $\chi^{2}=4.799$ \\
\hline $31-40$ & $21(6.9)$ & $47(15.4)$ & $68(22.2)$ & $\stackrel{\imath}{P}=0.309$ \\
\hline $41-50$ & $2(0.7)$ & $9(2.9)$ & $11(3.6)$ & Cramer's V $=0.309$ \\
\hline 51 and above & $0(0.0)$ & $1(0.3)$ & $1(0.3)$ & \\
\hline Total & $66(21.6)$ & $240(78.4)$ & $306(100)$ & \\
\hline \multicolumn{5}{|l|}{ Marital status } \\
\hline Single & 35 (11.4) & $143(46.7)$ & $178(58.2)$ & $\chi^{2}=3.121$ \\
\hline Married & $27(8.8)$ & $73(23.9)$ & $100(32.7)$ & $\stackrel{P}{P}=0.373$ \\
\hline Divorced/separated & $3(1.0)$ & $29(6.5)$ & $23(7.5)$ & Cramer's V $=0.373$ \\
\hline Widow/widower & $1(0.3)$ & $4(1.3)$ & $5(1.6)$ & \\
\hline Total & $66(21.6)$ & $240(78.4)$ & $306(100)$ & \\
\hline \multicolumn{5}{|l|}{ Educational level } \\
\hline No formal education & $4(1.3)$ & $19(6.2)$ & $23(7.5)$ & $\chi^{2}=4.229$ \\
\hline JHS & $7(2.3)$ & $30(9.8)$ & $37(12.1)$ & $\stackrel{P}{P}=0.376$ \\
\hline SHS & $26(8.5)$ & $98(32.0)$ & $124(40.5)$ & Cramer's V $=0.376$ \\
\hline Vocational & $15(4.9)$ & $65(21.2)$ & $80(26.1)$ & \\
\hline Tertiary & $14(4.6)$ & $28(9.2)$ & $42(13.7)$ & \\
\hline Total & $66(21.6)$ & $240(78.4)$ & $306(100)$ & \\
\hline \multicolumn{5}{|l|}{ Residence } \\
\hline Rural & $24(7.8)$ & $46(15.0)$ & $70(22.9)$ & $\chi^{2}=8.677$ \\
\hline Urban & $42(13.7)$ & $194(63.4)$ & $236(77.1)$ & $P=0.03$ \\
\hline Total & $66(21.6)$ & $240(78.4)$ & $306(100)$ & Cramer's V $=0.003$ \\
\hline \multicolumn{5}{|l|}{ Sector of work } \\
\hline Restaurant & $40(13.1)$ & $142(46.4)$ & $182(59.5)$ & $\chi^{2}=5.553$ \\
\hline Fast food & $16(5.2)$ & $55(18.0)$ & $71(23.1)$ & $\stackrel{P}{P}=0.136$ \\
\hline Hotel & $10(3.3)$ & $26(8.5)$ & $36(11.8)$ & Cramer's V $=0.136$ \\
\hline Chop bar & $0(0.0)$ & $17(5.6)$ & $17(5.60)$ & \\
\hline Total & $66(21.6)$ & $240(78.4)$ & $306(100)$ & \\
\hline
\end{tabular}

TABLe 2: Respondents knowledge of food safety and HACCP.

\begin{tabular}{lcccccc}
\hline \multirow{2}{*}{ Category } & \multicolumn{3}{c}{ Knowledge about food safety } & \multicolumn{3}{c}{ Knowledge about HACCP } \\
& Yes $N(\%)$ & No $N(\%)$ & Not sure $N(\%)$ & Yes $N(\%)$ & No $N(\%)$ & Not sure $N(\%)$ \\
\hline Restaurants & $162(52.9)$ & $10(3.3)$ & $10(3.3)$ & $65(21.2)$ & $81(26.5)$ & $36(11.8)$ \\
Fast food & $62(20.3)$ & $5(1.6)$ & $4(1.3)$ & $16(5.2)$ & $13(4.2)$ & $7(2.3)$ \\
Hotels & $35(11.4)$ & $1(0.3)$ & $0(0.0)$ & $0(0.0)$ & $17(5.6)$ & $0(0.0)$ \\
Chop bars & $6(2.0)$ & $10(3.3)$ & $1(0.3)$ & $97(31.7)$ & $149(48.7)$ & $60(19.6)$ \\
\hline
\end{tabular}

3.3. Food Safety Attitudes and Practices. Table 3 presents respondents' attitudes to food safety. The majority (78.4\%) of the respondents stated that food safety is a major issue of public concern. However, $62.7 \%$ of the participants indicated that cross-contamination is key to food safety. Moreover, $95.8 \%$ of respondents indicated that hand washing is important in practicing food safety. Interestingly, the majority of respondents $(75.8 \%)$ indicated that food 


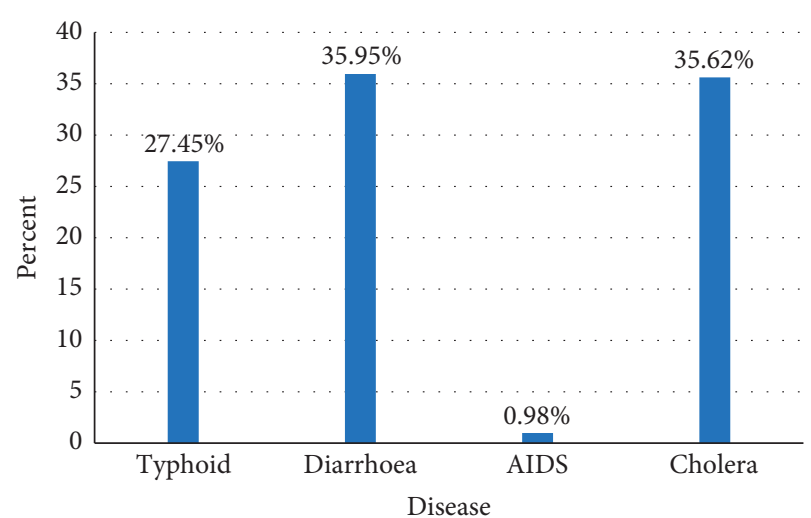

Figure 2: Diseases associated with improper handling of food.

safety practices are not the responsibility of the kitchen staff only. Also $69.3 \%$ of the respondents stated that food safety training is a necessity or need.

Table 4 presents respondent workplace hygiene practices. Most respondents $(80.1 \%)$ indicated that they wash their hands after handling raw meat when cooking. The majority $(93.8 \%)$ of respondents also indicated that they wash their hands after using the wash room. It can also be observed form the table that most (65.7\%) of the respondents maintain standard temperatures for food storage. Moreover, $36.6 \%$ stated that they do not use a checklist in cleaning at their workplaces. Lastly, $69.6 \%$ stated that they separate fresh produce from ready-to-eat food in refrigerator.

Figure 3 shows the respondent annual medical screening. From the analysis, the majority of the respondents $(65.69 \%)$ indicated that they undergo annual medical screening at their workplaces. However, $32.35 \%$ confirmed that there is no annual medical screening for food handlers at their workplaces. However, $1.96 \%$ indicated that sometimes they undergo annual medical screening at their workplaces.

3.4. Barriers to Food Safety. Table 5 illustrates the respondent views of barriers to food safety. Lack of training/education was identified by the majority (63.1\%) of the respondents as a major barrier to food safety. This was followed by lack of law enforcement (20\%), lack of time (12.7\%), and inadequate water supply (3.5\%). However, it is worth noting that $0.5 \%$ of the respondents took the view that laziness is also a barrier to food safety. Interestingly, there was no observed variance in views expressed by males and females.

Table 6 shows the correlation between food safety practice and selected demographic variables.

From the table, it can be observed that there is positive correlation between food safety practice and the following variables: ethnicity, marital status, and type of residence. However, there is a negative correlation between food safety practice and the following variables: age and education level. No correlation was observed between food safety practice and the following variables: religion, gender, and work sector.

\section{Discussion}

The aim of this paper is to evaluate food safety knowledge and practices among food handlers in the Ghanaian hospitality sector. This is aimed at informing public policy and improving food safety. Food safety is critical for public health and economic development. It is also important for the growth and patronage of the hospitality sector [3]. Indeed, more than 200 diseases are transmitted by food and the vast majority of the population will contract a foodborne disease at some point in their lifetime [15].

4.1. Respondents Knowledge of Food Safety and HACCP. The respondents' knowledge of food safety was obtained through the assessment of food safety principles (Table 2) and diseases associated with improper handling of food (Figure 2).

The assessment of respondents' knowledge on food safety practices and hazard analysis and control points (HACCP) was carried out in this study. HACCP is an internationally certified strategy towards food management practices. Hence, its inclusion in the study was vital. In the present study, with exception of food handlers who operate in chop bars, we found that a high proportion of respondents were knowledgeable about food safety practices. Food handlers in chop bars had the least knowledge of food safety. The general high knowledge of respondents observed in this study is in agreement with similar studies in the subregion $[16,17]$. It is also worth noting that the low knowledge of food safety practices observed among chop bar food handlers corroborates with work done by Abankwah [18] that suggested that chop bar food handlers having low knowledge of food safety practices was due to low education level and training.

However, proportionally, the majority of the respondents in various food sectors had no knowledge about HACCP which is compelling because, according to Pal et al. [19], this food management practice is flexible and can be utilized across the entire food chain. Thus, its adoption in the food industry should not be complicated. The observations made in this study aggress with a number of related studies. Tuncer and Akoglu [20] found that less than half of participants (48.70\%) from hotel kitchens in Turkey knew about HACCP. Similar results were obtained from the study of Ulusoy and Çolakoğlu [21]. Furthermore, studies from Ireland by Bolton et al. [22] and from Brazil by Rebouças et al. [23] observed that nearly $35.0 \%$ of the participants knew what the HACCP means.

There were interesting observations from the evaluation of the respondent's knowledge of diseases associated with unsafe handling of food (Figure 2). Generally, the most commonly reported foodborne diseases include diarrhea, nausea, vomiting, and stomach cramps [24]. In this study, $35.9 \%$ of the respondents mentioned diarrhea as a foodborne disease, with $27.45 \%$ and $35.62 \%$ pointing to typhoid and cholera, respectively. It is worth noting that, though our observation shows a moderate knowledge level of foodborne diseases, it appears to be at a low rate compared to similar 
TABLE 3: Respondents' attitudes to food safety.

\begin{tabular}{|c|c|c|c|}
\hline Variable & Yes & No & Not sure \\
\hline Is food safety a major issue of public concern? & $240(78.4)$ & $8(2.6)$ & $58(19.0)$ \\
\hline Must fresh produce be washed before cooking? & $294(96.1)$ & $7(2.3)$ & $5(1.6)$ \\
\hline Is cross-contamination key to food safety? & $192(62.7)$ & $99(32.4)$ & $15(4.9)$ \\
\hline Is hand washing important in practicing food safety? & $293(95.8)$ & $8(2.6)$ & $5(1.6)$ \\
\hline Is food safety important to your company's daily operation? & $26(85.6)$ & $9(2.9)$ & $35(11.4)$ \\
\hline Can food safety practice increase your company revenue? & $231(75.5)$ & $28(9.2)$ & $47(15.4)$ \\
\hline Is food safety practice the responsibility of kitchen staff only? & $74(24.2)$ & $232(75.8)$ & $0(0)$ \\
\hline Should one use separate surfaces for raw and cooked food? & $215(70.3)$ & $91(29.7)$ & $0(0)$ \\
\hline Is food safety training necessity or need? & $212(69.3)$ & $61(19.9)$ & $33(10.80)$ \\
\hline
\end{tabular}

TABLE 4: Respondent workplace hygiene practices.

\begin{tabular}{lccc}
\hline Variable & Yes $(N(\%))$ & No $(N(\%))$ & Sometimes $(N(\%))$ \\
\hline Do you wash your hands after handling raw meat when cooking? & $245(80.1)$ & $8(2.6)$ & $53(17.3)$ \\
Do you wash your hands after using the wash room? & $287(93.8)$ & $8(2.6)$ & $11(3.6)$ \\
Do you maintain standard temperatures for food storage? & $201(65.7)$ & $99(32.4)$ & $6(2.00)$ \\
Are dustbins covered all the time? & $206(67.3)$ & $53(17.3)$ & $47(15.4)$ \\
Do you use a checklist in cleaning? & $99(32.4)$ & $112(36.6)$ & $95(31.0)$ \\
Do you separate fresh produce from ready-to-eat food in refrigerator? & $213(69.6)$ & $51(16.7)$ & $42(13.7)$ \\
\hline
\end{tabular}

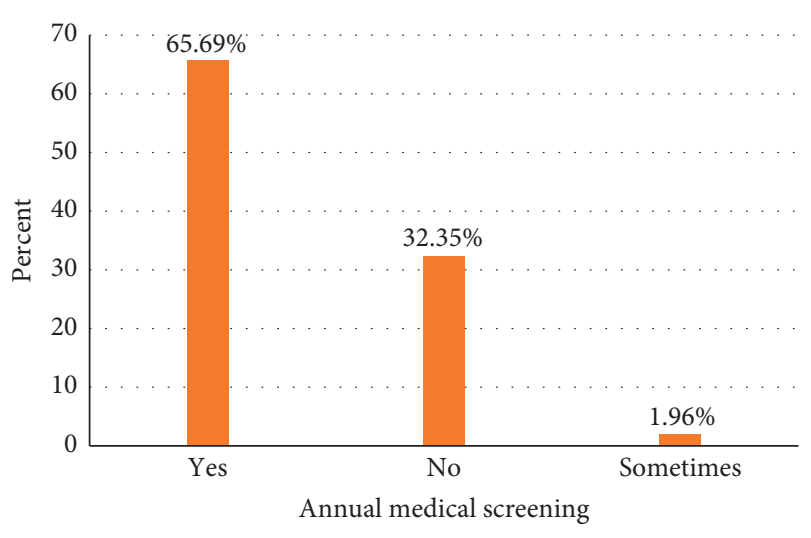

Figure 3: Annual food vendor medical screening

studies. For example, Osaili et al. [25, 26] reported that $90 \%$ of food service staff working in the hospitals and restaurants had knowledge about the most common symptoms of foodborne diseases such as diarrhea. Similarly, Jianu and Chis [27] indicated that the majority of food handlers (77\%) in Romania knew about foodborne diseases. It is important to know that diarrhea is the most common foodborne disease that runs through many studies $[25,26,28]$. Osaili et al. [26] clarified that this observation was as a result of the emphasis placed on diarrhea by the main stream media. Quite interestingly, about 1\% of the responses listed HIV/ AIDS as another possible food born disease. This calls for education for these food handlers. Though having knowledge does not directly translate into safe food practices, it is a constant reminder of how devastating contaminated food can be to customers. This may also initiate the influence of positive attitudes and practices towards food safety.
4.2. Food Safety Attitudes and Practices. The study examined food safety practices of the respondents. This was achieved through assessment of respondents' attitudes to food safety (Table 3), respondents' workplace hygiene practices ( Table 4), and annual food vendor medical screening (Figure 3 ). Review of the literature shows that several studies related to food safety in the hospitality industry have revealed that sufficient knowledge and positive attitudes do not necessarily translate into good practices $[9,29,30]$. Data from Table 5 and Figure 3 show the practice level of respondents with regard to food safety. Generally, the average practice score of the respondents was $68.2 \%$ which is satisfactory. Specifically, respondents revealed poor practice scores regarding the use of checklist in cleaning with only $32.4 \%$ indicating that they practice the technique. However, checklist is very important in ensuring that there is standardization of cleaning and also that certain areas or items are not skipped during disinfection or decontamination in food industries and sectors.

Furthermore, $67.3 \%$ of the respondents reported covering dustbins all the time. It was, however, intriguing that about $17 \%$ of the vendors revealed that their workplace bins are not covered at all times. Uncovered bins in food service centers are an indicator of poor hygiene. Poor hygiene which eventually results in food contamination is known to be a major cause of cholera [28, 29]. Moreover, covering bins at all times is of great essence in all food sectors within Ghana's hospitality industry, in order to avoid cholera and related foodborne disease outbreaks $[3,31]$.

It was also observed from this study that the majority (95.8\%) of the respondents indicated that hand washing is important in practicing food safety. This observation is similar to that of Tan et al. [32] and Reboucas et al. [23] who 
TABLE 5: Barriers to food safety.

\begin{tabular}{lccc}
\hline Variables & $\begin{array}{c}\text { Male } N \\
(\%)\end{array}$ & $\begin{array}{c}\text { Female } N \\
(\%)\end{array}$ & $\begin{array}{c}\text { Total } N \\
(\%)\end{array}$ \\
\hline $\begin{array}{l}\text { Lack of training/ } \\
\text { education }\end{array}$ & $114(37.1)$ & $79(26)$ & $193(63.1)$ \\
Inadequate water supply & $9(2.8)$ & $2(0.7)$ & $11(3.5)$ \\
Lack of time & $36(11.7)$ & $39(1.1)$ & $39(12.7)$ \\
Laziness & $2(0.5)$ & $0(0.0)$ & $2(0.5)$ \\
Lack of law enforcement & $56(18.2)$ & $6(1.8)$ & $61(20)$ \\
\hline
\end{tabular}

TABLE 6: Correlation between food safety practice and selected variables.

\begin{tabular}{lcc}
\hline Sr. no. & Variables & Pearson correlation \\
\hline 1 & Age & -0.004 \\
2 & Gender & $0.148^{* *}$ \\
3 & Religion & $0.182^{* *}$ \\
4 & Ethnicity & 0.040 \\
5 & Educational level & -0.003 \\
6 & Work sector & $0.099^{*}$ \\
7 & Marital status & 0.005 \\
8 & Residence & 0.043 \\
\hline
\end{tabular}

* Significant correlation at $P<0.05$ level (2-tailed). ${ }^{* *}$ Significant correlation at $P<0.01$ level (2-tailed).

indicated that the majority of food handlers had inadequate knowledge about hand washing.

The study also sought to investigate annual medical screening of the food handlers at their workplaces as a criterion of food safety practice. Annual medical screening of the food handlers is very important in preventing people with communicable diseases from handling food [33]. In fact, local laws such as Section 286 of the Criminal Code (Amendment) Act, 2003 (Act 646), make it mandatory for all food handlers and vendors to undergo medical examinations to prevent transmission of communicable diseases from food handlers to customers [33]. In the study, we found that $65.7 \%$ of the respondents indicated that medical screening was carried out at the worksites annually. However, $32.4 \%$ of the responding food vendors indicated that such an activity was not carried out at all at their workplaces. This might be a suggestion that appropriate law enforcers are not enforcing exiting laws.

It is worth noting that attitudes of food vendors or handlers towards food safety practices strongly influence the occurrence of foodborne disease illness [9]. Hence, there is a major linkage between positive attitudes and implementation of safe food handling practices. In the present study, the overall average attitude score of respondents obtained (Table 4) was $78.8 \%$ which signified that the majority of the responding food vendors had positive attitudes towards food safety. Positive attitudes of food vendors towards food safety/handling practices have been reported [9, 34-36]. However, these attitudes do not necessarily translate into practice, yet they give an indication of knowledge and mindsets. The present finding is an indicator that food vendors in Ghana are gradually developing positive mindsets and attitudes towards food safety issues which could reduce cases of foodborne illness in the country.
4.3. Barriers to Food Safety. The study also obtained the opinions of the food handlers regarding the barriers to food safety. Generally, respondents indicated lack of training, inadequate water supply, laziness, and lack of laws enforcement as barriers to food safety in Ghana's hospitality industry. Several researches have shown that barriers to food safety can vary in different regions which could affect direction of policymaking and stakeholder decisions [37-39]. However, the main barrier identified was lack of training as it had $63.1 \%$ of total responses. Education and regular training of food service personnel are highly needed for improving food safety knowledge and practices [41]. In view of this, structures such as organization of practically and technically oriented courses/programs will be helpful to equip food vendors and handlers with best food safety practices in the hospitality industry. This suggestion was similarly revealed in [42].

4.4. Policy Implications and Conclusion. The study examined the food safety knowledge and practices among food handlers in the Ghanaian hospitality sector. The findings show that there is moderate level of awareness of food safety practices among the respondents. Also, most respondents who work in restaurants had knowledge about food safety principles. Lack of training and education was identified as a major barrier to food safety. Diarrhea, cholera, and typhoid were identified as major diseases associated with improper handling of food. A significant number of the respondents do not undergo annual medical screening at their workplaces. Further, there were significant associations between sociodemographic characteristics and food safety practices. Based on the findings of the study and the literature reviewed, four main policy recommendations are made.

First is the need for education and awareness of the harmful effects of poor food safety practices and more importantly strategies especially for proper food hygiene and safety.

The lack of food safety knowledge has implications for community, state, and national leaders. This could be achieved through public engagements, meetings, conferences, workshops, and training programs on the poor food safety procedures. The media, civil society organizations, youth, students, and health organizations should be actively involved in creating awareness of food safety.

Second is the need to have effective monitoring systems to assess food safety compliance among food handlers in the country. Without a monitoring system, it will be difficult to appreciate the seriousness and importance of the problem. This should also be complimented with strict law enforcement. This is key to ensuring that food safety rules and guidelines are effectively implemented in the food industry.

Finally, many bottom-up studies are needed to identify and improve strategies for enhancing food safety. This study is one of the bottom-up studies to assess the extent of awareness and practices of food handlers in the hospitality sector. Further studies are needed to deepen our understanding of food safety issues and more importantly practical strategies for promoting food safety practices [40]. 


\section{Data Availability}

The edge service data used to support the findings of this study are included within the article.

\section{Conflicts of Interest}

The authors declare that they have no conflicts of interest.

\section{References}

[1] A. Sulemana, "An evaluation of government service quality in a growing democracy: national democratic congress in Ghana (2012-2016)," Journal of Nonprofit \& Public Sector Marketing, vol. 31, no. 5, pp. 464-485, 2019.

[2] E. A. Adu-Ampong, "Tourism and national economic development planning in Ghana, 1964-2014," International Development Planning Review, vol. 40, no. 1, pp. 75-95, 2018.

[3] J. Dzeagu-Kudjodji, S. Adjibolosoo, and D. Otoo-Arthur, "Promoting indigenous dishes in luxurious hotels in the hospitality industry: the case of three selected hotels in the Accra metropolis of greater Accra region," European Journal of Research and Reflection in Management Sciences, vol. 7, no. 4, 2019.

[4] S. Klimpel, T. Kuhn, J. Münster, D. D. Dörge, R. Klapper, and J. Kochmann, Food Safety Considerations. InParasites of Marine Fish and Cephalopods, pp. 149-155, Springer, Berlin, Germany, 2019.

[5] G. K. Amoako, E. K. Neequaye, S. G. Kutu-Adu, L. D. Caesar, and K. S. Ofori, "Relationship marketing and customer satisfaction in the Ghanaian hospitality industry," Journal of Hospitality and Tourism Insights, vol. 4, 2019.

[6] C. J. Lagerkvist, F. Amuakwa-Mensah, and J. Tei Mensah, "How consumer confidence in food safety practices along the food supply chain determines food handling practices: evidence from Ghana," Food Control, vol. 93, pp. 265-273, 2018.

[7] P. F. Ababio and P. Lovatt, "A review on food safety and food hygiene studies in Ghana," Food Control, vol. 47, pp. 92-97, 2015.

[8] A. Alagiyawanna, "AMAAP P S A. Effects of multiple interventions on the hygienic standards of food handling establishments," MOJ Public Health, vol. 7, no. 6, pp. 297-302, 2018.

[9] F. Akabanda, E. H. Hlortsi, and J. Owusu-Kwarteng, "Food safety knowledge, attitudes and practices of institutional foodhandlers in Ghana," BMC Public Health, vol. 17, no. 1, p. 40, 2017.

[10] O. P. Aovare, "Food Vending Hygiene Practices in the Bolgatanga Municipality of the Upper East Region," Doctoral dissertation, University of Cape Coast, Cape Coast, Ghana, 2017.

[11] I. Monney, D. Agyei, B. S. Ewoenam, C. Priscilla, and S. Nyaw, "Food hygiene and safety practices among street food vendors: an assessment of compliance, institutional and legislative framework in Ghana," Food and Public Health, vol. 4, no. 6, pp. 306-315, 2014.

[12] S. Apanga, J. Addah, and D. R. Sey, "Food safety knowledge and practice of street food vendors in rural Northern Ghana," Food and Public Health, vol. 4, no. 3, pp. 99-103, 2014.

[13] S. T. Odonkor, T. Adom, R. Boatin, D. Bansa, and C. J. Odonkor, "Evaluation of hygiene practices among street food vendors in Accra metropolis, Ghana," Elixir Online Journal, vol. 41, pp. 5807-5811, 2011.
[14] R. L. Miller and J. D. Brewer, Eds., The AZ of Social Research: A Dictionary of Key Social Science Research Concepts, Sage, Thousand Oaks, CA, USA, 2003.

[15] C. Ruiz-Capillas and A. Herrero, "Impact of biogenic amines on food quality and safety," Foods, vol. 8, no. 2, p. 62, 2019.

[16] R. Addo-Tham, E. Appiah-Brempong, H. Vampere, E. Acquah-Gyan, and A. Gyimah Akwasi, "Knowledge on food safety and food-handling practices of street food vendors in ejisu-juaben municipality of Ghana," Advances in Public Health, vol. 2020, Article ID 4579573, 7 pages, 2020.

[17] M. Y. Madaki and M. Bavorova, "Food safety knowledge of food vendors of higher educational institutions in Bauchi state, Nigeria," Food Control, vol. 106, p. 106703, 2019.

[18] a Abankwah, "Effect of 'chop-bar'foods and its health implications on consumers in Kumasi metropolis, Ghana," International Journal of Education and Research, vol. 6, 2018.

[19] M. Pal, W. Gebregabiher, and R. K. Singh, "The role of hazard analysis critical control point in food safety," Beverage \& Food World, vol. 43, no. 4, p. 34, 2016.

[20] T. Tuncer and A. Akoğlu, "Food safety knowledge of food handlers working in hotel kitchens in Turkey," Food and Health, vol. 6, no. 2, pp. 67-89, 2020.

[21] B. Ulusoy and N. Çolakoğlu, "A survey on determination of HACCP knowledge of food handlers in Istanbul food businesses," Acta Alimentaria, vol. 44, no. 3, pp. 342-348, 2015.

[22] D. J. Bolton, A. Meally, I. S. Blair, D. A. McDowell, and C. Cowan, "Food safety knowledge of head chefs and catering managers in Ireland," Food Control, vol. 19, no. 3, pp. 291-300, 2008.

[23] L. T. Rebouças, L. B. Santiago, L. S. Martins, A. C. Rios Menezes, M. d. P. N. Araújo, and R. C. d. C. Almeida, "Food safety knowledge and practices of food handlers, head chefs and managers in hotels' restaurants of Salvador, Brazil," Food Control, vol. 73, pp. 372-381, 2017.

[24] CDC (The Centers for Disease Control and Prevention of the USA), Foodborne Illnesses and Germs, Atlanta, GE, USA, 2018, https://www.cdc.gov/foodsafety/foodborne-germs.html/.

[25] T. M. Osaili, B. A. Obeidat, W. A. Hajeer, and A. A. AlNabulsi, "Food safety knowledge among food service staff in hospitals in Jordan," Food Control, vol. 78, pp. 279-285, 2017.

[26] T. M. Osaili, B. A. Obeidat, D. O. Abu Jamous, and H. A. Bawadi, "Food safety knowledge and practices among college female students in North of Jordan," Food Control, vol. 22, no. 2, pp. 269-276, 2011.

[27] C. Jianu and C. Chiş, "Study on the hygiene knowledge of food handlers working in small and medium-sized companies in Western Romania," Food Control, vol. 26, no. 1, pp. 151-156, 2012.

[28] B. Tokuç, G. Ekuklu, U. Berberoğlu, E. Bilge, and H. Dedeler, "Knowledge, attitudes and self-reported practices of food service staff regarding food hygiene in Edirne, Turkey," Food Control, vol. 20, no. 6, pp. 565-568, 2009.

[29] U. M. Asmawi, A. A. Norehan, K. Salikin, N. A. Rosdi, N. A. Munir, and N. B. Basri, "An assessment of knowledge, attitudes and practices in food safety among food handlers engaged in food courts," Current Research in Nutrition and Food Science Journal, vol. 6, no. 2, pp. 346-353, 2018.

[30] C. Bou-Mitri, D. Mahmoud, N. El Gerges, and M. A. Jaoude, "Food safety knowledge, attitudes and practices of food handlers in Lebanese hospitals: a cross-sectional study," Food Control, vol. 94, pp. 78-84, 2018.

[31] E. K. Dzotsi, "Addressing the cholera epidemic," Retrieved, vol. 12, pp. 4-15, 2014.

[32] S. L. Tan, F. A. Bakar, M. S. Abdul Karim, H. Y. Lee, and N. A. Mahyudin, "Hand hygiene knowledge, attitudes and 
practices among food handlers at primary schools in Hulu Langat district, Selangor (Malaysia)," Food Control, vol. 34, no. 2, pp. 428-435, 2013.

[33] Section 286, The Criminal Code of Ghana (Amendment) Act, 2003 (Act 646), http://www.refworld.org/docid/44bf823a4. html, 2013.

[34] D. Al-Kandari, J. Al-abdeen, and J. Sidhu, "Food safety knowledge, attitudes and practices of food handlers in restaurants in Kuwait," Food Control, vol. 103, pp. 103-110, 2019.

[35] A. L. Dora-Liyana, N. A. Mahyudin, M. Rashedi, A. A. IsmailFitry, and H. Rasiyuddin, "Food safety and hygiene knowledge, attitude and practices among food handlers at boarding schools in the northern region of Malaysia," Social Sciences, vol. 8, no. 17, pp. 238-266, 2018.

[36] N. A. Al-Shabib, S. H. Mosilhey, and F. M. Husain, "Crosssectional study on food safety knowledge, attitude and practices of male food handlers employed in restaurants of King Saud University, Saudi Arabia," Food Control, vol. 59, pp. 212-217, 2016.

[37] R. Nayak and P. Waterson, "The Assessment of Food Safety Culture: an investigation of current challenges, barriers and future opportunities within the food industry," Food Control, vol. 73, pp. 1114-1123, 2017.

[38] I. Young and L. Waddell, "Barriers and facilitators to safe food handling among consumers: a systematic review and thematic synthesis of qualitative research studies," PloS One, vol. 11, no. 12, Article ID e0167695, 2016.

[39] J. Kang, J. Jun, and S. W. Arendt, “Understanding customers' healthy food choices at casual dining restaurants: using the Value-Attitude-Behavior model," International Journal of Hospitality Management, vol. 48, pp. 12-21, 2015.

[40] C. O. Medeiros, S. B. Cavalli, E. Salay, and R. P. C. Proença, "Assessment of the methodological strategies adopted by food safety training programmes for food service workers: a systematic review," Food Control, vol. 22, no. 8, pp. 1136-1144, 2011.

[41] N. A. Alqurashi, A. Priyadarshini, and A. K. Jaiswal, "Evaluating food safety knowledge and practices among foodservice staff in Al madinah hospitals, Saudi Arabia," Safety, vol. 5, no. 1, p. 9, 2019.

[42] A. P. Kunadu, D. B. Ofosu, E. Aboagye, and K. Tano-Debrah, "Food safety knowledge, attitudes and self-reported practices of food handlers in institutional foodservice in Accra, Ghana," Food Control, vol. 69, pp. 324-330, 2016. 